\section{Análise da produção ambulatorial em municípios com e sem centros de especialidades odontológicas no Brasil em 2010}

\author{
Analysis of outpatient care in Brazilian \\ municipalities with and without \\ specialized dental clinics, 2010
}

\author{
Análisis de la producción ambulatoria en \\ municipios con y sin centros de especialidades \\ dentales en Brasil, 2010
}

Roger Keller Celeste

Flávio Renato Reis de Moura 2

Carolina Paiva Santos 1

Maximiano Ferreira Tovo ${ }^{2}$

\author{
${ }^{1}$ Faculdade de Odontologia, \\ Universidade Federal do Rio \\ Grande do Sul, Porto Alegre, \\ Brasil. \\ 2 Universidade Luterana do \\ Brasil, Canoas, Brasil. \\ Correspondência \\ R. K. Celeste \\ Departamento de Odontologia \\ Preventiva e Social, Faculdade \\ de Odontologia, Universidade \\ Federal do Rio Grande do Sul. \\ Rua Ramiro Barcelos 2492 \\ Porto Alegre, RS 90035-003, \\ Brasil. \\ roger.keller@ufrgs.br
}

Abstract

The aim of this study was to describe the production of specialized outpatient dental services and associated factors in Brazilian municipalities (counties) with and without Specialized Dental Clinics (SDC). Data were collected from the following sources: Information Technology Department of the Unified National Health System, Human Development Atlas for Brazil, and Brazilian Institute of Geography and Statistics. Zeroinflated negative binomial regression was used to fit rates of endodontic procedures, primary care, periodontics, and surgery. After controlling for socio-demographic factors, municipalities with SDC showed higher rates for the abovementioned procedures than those without, except for primary care. In the final model, including service structure variables, municipalities with type III SDC showed a rate of endodontic procedures that was 2.08 times higher (95\% CI: 1.26; 3.44) than those without. Among the structural variables, municipalities with higher health expenditures and more dentists in the Unified $\mathrm{Na}$ tional Health System showed consistently higher rates for all types of procedures. The SDC appear to have a positive effect on the local production of specialized procedures (especially endodontic), an effect not explained by structural variables.

Dental Health Services; Health Policy; Secondary Care; Ecological Studies

\section{Resumo}

O objetivo foi descrever a produção de serviços especializados em municípios brasileiros com e sem Centros de Especialidades Odontológicas (CEO) e estudar fatores associados. Para a coleta de dados, foram consultados: o Departamento de Informática do SUS, dados do Atlas de Desenvolvimento Humano do Brasil e do Instituto Brasileiro de Geografia e Estatística. Utilizou-se regressão binominal negativa inflada de zeros para modelar taxas de procedimentos de endodontia, atenção básica, periodontia e cirurgia. Após controle por fatores sociodemográficos, os municípios com CEO apresentaram taxas maiores do que os sem, exceto para taxas de atenção básica. No modelo final, com variáveis de estrutura dos serviços, os municípios com CEO do tipo III tiveram uma taxa de procedimentos de endodontia 2,08 (IC95\%: 1,26; 3,44) vezes maior que sem CEO. Das variáveis de estrutura, mais gastos em saúde e maiores taxas de dentistas no SUS estavam consistentemente associados a maiores taxas de quaisquer procedimentos. Os CEO parecem ter um efeito positivo na produção municipal de procedimentos especializados, particularmente para a taxa de procedimentos de endodontia, e esse efeito não é explicado por variáveis de estrutura.

Serviços de Saúde Bucal; Políticas de Saúde; Atenção Secundária à Saúde; Estudos Ecológicos 


\section{Introdução}

Os Centros de Especialidades Odontológicas (CEO) são unidades de referência para atenção secundária com a missão de realizar procedimentos de periodontia, endodontia, cirurgia oral menor e atendimento aos pacientes com necessidades especiais 1. Os CEO representam uma das propostas da Política Nacional de Saúde Bucal 2, estabelecida em 2004, e que está no conjunto das reformas política, sanitária e orçamentária da atenção primária e secundária ${ }^{3}$. Com os CEO, vislumbra-se aumentar o acesso da população a serviços especializados e atingir integralidade da atenção dos serviços odontológicos providos pelo Sistema Único de Saúde (SUS) 2,4.

São encontrados na literatura alguns estudos avaliando os CEO em dimensões de integralidade da atenção, cumprimento de metas, taxa de utilização dos serviços e satisfação dos usuários 5,6,7,8,9. Em relação à integralidade, usuários cobertos pela Estratégia Saúde da Família (ESF) apresentaram 1,56 vez mais probabilidade de obter atendimento nos CEO, especialmente para procedimentos endodônticos, porém, se o paciente era referenciado pela atenção básica, tinha 5,9 vezes mais probabilidade de atendimento especializado quando comparado aos pacientes de livre demanda ${ }^{6}$. Em relação ao cumprimento das metas, estudos mostraram que municípios com baixo Índice de Desenvolvimento Humano (IDH) e de pequeno porte populacional tinham menos chances de atingir as metas preconizadas pelo Ministério da Saúde 5,9. Ainda, em um trabalho de caráter quantitativo e qualitativo no Estado da Bahia, descreveu-se que houve cumprimento das metas nas especialidades de periodontia e endodontia; no entanto, destaca-se a subutilização dos serviços ${ }^{8}$. Hipóteses para a subutilização dos serviços estão relacionadas com o não cumprimento da carga horária de trabalho pelos profissionais, não reposição de pacientes que faltam às consultas, e dificuldades no sistema de referência ${ }^{8}$. Por fim, usuários assistidos nos CEO avaliaram positivamente as dimensões de relações humanas, resolubilidade e infraestrutura das unidades, contudo a dimensão acesso foi avaliada negativamente, pois $52,5 \%$ dos usuários responderam ser difícil conseguir vaga para atendimento 7 .

Apesar dos investimentos do Ministério da Saúde nas implantações de CEO, ainda não há estudos em nível nacional sobre a produtividade dos municípios com os centros. Poderia se esperar que a implantação de CEO aumentasse a produção municipal total de procedimentos especializados na área da odontologia, em vez de haver apenas uma migração deles da aten- ção básica para a especializada. Tal aumento se daria porque a implantação de CEO implicaria mais profissionais na rede, sem que a atenção básica deixasse de realizar eventuais procedimentos especializados que já estavam em sua rotina. Há também a possibilidade de a atenção especializada realizar procedimentos de atenção básica, quando ela não estiver bem organizada 6. Por fim, não se conhece o papel, na produção de serviços odontológicos especializados, de políticas municipais como: o aumento de oferta de profissionais, equipamentos e investimentos financeiros. Assim, o objetivo do presente estudo foi descrever a produção de serviços especializados em municípios com e sem CEO e estimar a associação deles com alguns fatores que podem influenciar nessa produção.

\section{Métodos}

Delineamento do estudo e fontes de informação

Este foi um estudo ecológico que incluiu todos os municípios brasileiros no ano de 2010. Foram utilizados dados secundários de vários sistemas de informação do Departamento de Informática do SUS (DATASUS; http://www.datasus.gov. br): Cadastro Nacional de Estabelecimentos em Saúde (CNES); Sistema de Informações Ambulatoriais (SIA-SUS); Sistema de Informações sobre Orçamentos Públicos em Saúde (SIOPS); Sistema de Informações em Atenção Básica (SIAB); Agência Nacional de Saúde Suplementar - Sistema de Informações de Beneficiários (ANS-SIB). Também foram extraídos dados do Atlas de Desenvolvimento Humano do Brasil (ATLAS) 10 e Instituto Brasileiro de Geografia e Estatística (IBGE; http://www.ibge.gov.br). A coleta de dados ocorreu entre os meses de março e dezembro de 2011 por um único pesquisador previamente treinado.

\section{Variáveis em estudo}

O estudo teve como variáveis de desfecho os quatro tipos de procedimentos odontológicos realizados nos CEO, conforme a Portaria no 2.898: procedimentos básicos, procedimentos de periodontia, procedimentos de endodontia e procedimentos de cirurgia oral 11. Os números de procedimentos realizados pelos CEOs de cada município foram obtidos do SIA-SUS. Foram calculadas as taxas de cada conjunto de procedimentos realizados no ano de 2010 por $10 \mathrm{mil}$ habitantes-ano para os municípios brasileiros.

Dados sobre o número e tipos de CEO registrados, sua data de habilitação/implantação e 
localização (municípios aos quais eles pertencem) foram obtidos do CNES. A classificação dos CEO seguiu a orientação da Portaria no 599 12, de forma a caracterizar o município, quando havia mais de um CEO, segundo aquele de maior tempo de instalação (porque a implantação ocorre em épocas diferentes) para a variável tempo de instalação, e de maior capacidade instalada (tipo III com 7 ou mais consultórios; tipo II constituído de 4-6 consultórios; e tipo I com 3 consultórios) para a variável tipo de CEO. Dados municipais sobre o tamanho populacional, número de habitantes por faixa etária, sexo e zona de residência foram obtidos do censo do IBGE para o ano de 2010. Logo, foram calculados, para cada município, os percentuais de: mulheres; habitantes entre 20 e 59 anos (adultos) e habitantes acima de 60 anos (idosos); e habitantes residindo na zona rural. Do IBGE também foi obtido o Produto Interno Bruto (PIB) municipal per capita de 2008 , ano mais próximo do desfecho. Do SIOPS foram obtidas as despesas totais em saúde per capita em Reais de 2010 (indicador 2.1). O IDH de 2000 e a renda média municipal dos indivíduos (ano 2000) foram obtidos do ATLAS. Dados sobre o número de pessoas cadastradas nas equipes de saúde bucal da ESF (ESB-ESF) para o ano de 2010 foram obtidos do SIAB para cálculo da cobertura percentual. Da ANS-SIB foi obtido o número de beneficiários de planos odontológicos privados de cada município para o ano de 2010, para posterior cálculo do percentual de beneficiários. Do CNES foi também obtido, para o ano de 2010, o número de equipos odontológicos completos e dentistas trabalhando no sistema único de saúde, com base nos quais foram calculadas taxas por 10 mil habitante-ano.

As variáveis $\mathrm{PIB}$ per capita, renda média, porte populacional do município, investimentos em saúde pública, cobertura de planos privados, cobertura populacional das ESB-ESF, equipos odontológicos, dentistas trabalhando no SUS foram categorizadas por quintis. Análises preliminares mostraram que algumas dessas relações não eram lineares.

\section{Análise estatística}

Foi apresentada análise bivariada da média das taxas de produção ambulatorial dos municípios que possuem CEO, sendo divididos em subgrupos conforme seu tipo de CEO, e dos municípios que nunca tiveram CEO. Também foram apresentados dados após a remoção dos municípios que não produziram nenhum procedimento e, portanto, deveriam não possuir capacidade instalada para produção de tais atendimentos.
Para análise multivariada, observou-se sobredispersão dos dados, confirmada com o teste deVoung $(\mathrm{p}<0,01)$. Além dessa característica, vários modelos apresentavam excesso de municípios com zero procedimento (teste qui-quadrado $\mathrm{p}<0,05)$. Desse modo, utilizaram-se modelos de regressão binomial negativa inflados de zeros (ZINB). Nesses casos, utiliza-se uma modelagem em dois processos distintos e simultâneos, com as mesmas ou diferentes variáveis em cada processo. Para o primeiro processo, ajustou-se um modelo para predizer se o município teria "zero procedimento" ou se teria algum procedimento notificado, sendo equivalente a uma regressão logística. No segundo processo, ajustaram-se modelos que explicavam a taxa de procedimentos dentre os municípios cujas taxas não foram preditas como zeros.

Para o primeiro processo, foram usados três preditores: porte populacional do município, taxa de dentistas trabalhando no SUS e a taxa de equipos em uso no SUS. Para o segundo processo, foram usados quatro modelos aninhados. O modelo 1 incluiu as variáveis relativas aos CEO: tipo e tempo de habilitação do CEO mais antigo e número de CEOs no município. Foram ajustados outros dois modelos com variáveis consideradas potenciais fatores de confusão. No modelo 2, foram adicionadas ao modelo anterior as variáveis demográficas que potencialmente alteram o uso de serviços: \% de mulheres, \% de idosos, \% de adultos, \% habitantes na zona rural e porte municipal. O modelo 3 constitui-se do acréscimo ao modelo prévio de variáveis socioeconômicas: IDH, PIB per capita e renda média dos habitantes. Finalmente, no modelo 4, foram adicionadas variáveis de serviços odontológicos que poderiam explicar diferenças entre os municípios: despesas totais em saúde per capita, \% de habitantes cadastrados por ESB-ESF, \% de beneficiários de planos odontológicos privados, taxa de equipos odontológicos disponíveis no SUS por 10 mil habitantes-ano e taxas de cirurgiõesdentistas no SUS por 10 mil habitantes-ano.

O estudo foi aprovado pela Comissão de Pesquisa de Odontologia da Universidade Federal do Rio Grande do Sul (protocolo no 21420). Em todas as análises, foi utilizado o programa estatístico Stata 11.2 (Stata Corp., College Station, Estados Unidos).

\section{Resultados}

Verificou-se que 4.851 municípios (87,2\% do total) não possuíam CEO cadastrados, e dos 713 municípios (12,8\%) com algum CEO cadastrado: $638(11,5 \%)$ possuíam apenas um CEO; os de- 
mais $75(1,3 \%)$ tinham entre 2 e 22 estabelecimentos. Os 713 municípios contabilizaram 863 centros divididos da seguinte forma: $320(37,1 \%)$ do tipo I, $454(52,6 \%)$ do tipo II e 89 (10,3\%) do tipo III. Os cinco municípios que mais possuíam CEO eram: Goiânia (Goiás) e Salvador (Bahia), com cinco CEOs; Belém (Pará) com seis; Rio de Janeiro com 16; e São Paulo com 22 CEOs. A média do tempo de habilitação em 2010 dos CEO foi de 3,4 anos (DP $\pm 1,9$ ), mas apenas $15,8 \%$ ( $n=$ 113) dos municípios possuíam CEO com tempo de habilitação de cinco anos ou mais.

Verificou-se que a taxa média de procedimentos básicos de periodontia, de endodontia e de cirurgia oral foram, respectivamente: 10.686/10 mil habitantes-ano; 75/10 mil habitantes-ano; 55/10 mil habitantes-ano; 293/10 mil habitantes-ano. Como características econômicas, os municípios brasileiros apresentaram a média de $\mathrm{R} \$ 10.373,90$ de $\mathrm{PIB} /$ per capita e a média de $\mathrm{R} \$$ 384,40 de despesa anual empenhada em saúde/ habitante (Tabela 1).

Na Tabela 2, constata-se que os municípios com centros produziram mais procedimentos, porém não sendo constante para todos os tipos de CEO, e para alguns procedimentos houve inversão dos resultados de produtividade. As taxas de procedimentos endodônticos são ilustrativas dessa inversão. A produção dos municípios sem CEO foi de 44,1 procedimentos/10 mil habitantes-ano, e a produção dos municípios com CEO tipo III foi de 91,9 procedimentos/10 mil habitantes-ano. Porém, quando são contabilizados apenas os municípios que realizaram ao menos um procedimento durante o ano de 2010, as mesmas taxas são, respectivamente, de 265,2 procedimentos/10 mil habitantes-ano e 94,3 procedimentos/10 mil habitantes-ano. Por fim, dentre os 713 municípios com CEO, cinco não realizam nenhum procedimento de atenção básica, 36 nenhum de endodontia, 41 nenhum de periodontia e sete nenhum de cirurgia oral menor.

A Tabela 3 apresenta os resultados dos modelos 1 a 3 e a Tabela 4, o modelo 4. O modelo 1 mostrou que os municípios com CEO tinham menores taxas de procedimentos básicos $(\mathrm{p}=$ 0,02 ). No modelo 2 , após o controle de fatores demográficos, foram encontradas maiores taxas de produção de procedimentos de cirurgia oral menor nos município com CEO quando comparados aos municípios sem CEO $(p=0,02)$. O modelo 3 mostrou que, após o controle de fatores socioeconômicos, os municípios com CEO tinham taxas de endodontias $(p=0,02)$, maiores taxas de cirurgia oral menor $(\mathrm{p}<0,01)$ e maiores taxas de procedimentos periodontais $(p=0,05)$. O modelo 3 mostrou que os municípios com CEO tinham taxas de procedimentos maiores que os municípios sem CEO, exceto para procedimentos básicos. Por exemplo, municípios com CEO tipo III tinham uma taxa de endodontia 1,89 (IC95\%: 1,15; 3,10) vez maior do que municípios sem CEO. Das variáveis socioeconômicas, IDH e PIB per capita não foram significativas ( $p>0,05)$, mas os municípios com indivíduos mais ricos tiveram maiores taxas para qualquer um dos procedimentos $(\mathrm{p}<0,01)$. No modelo 4 (Tabela 5), observa-se que apenas para procedimentos endodônticos permaneceu significativa $(\mathrm{p}<0,05)$ a diferença entre municípios com e sem CEO. Parte do "efeito CEO" na produtividade de procedimentos de cirurgia e periodontia foi explicada por variáveis relacionadas aos serviços de saúde. Procedimentos de atenção básica permaneceram não significantes ( $\mathrm{p}>0,05)$.

Um mesmo conjunto de variáveis foi usado em todas as regressões para predizer o processo de inflação de zeros. Os resultados desse modelo são idênticos para qualquer tipo de procedimento, assim foi apresentado apenas uma vez em tabela separada (Tabela 4). Somente para procedimentos básicos o número de equipos odontológicos foi uma variável estatisticamente significativa, a presença de mais equipos resultava em menores chances de o município ter uma taxa igual a zero para esse tipo de procedimento. $\mathrm{O}$ número de dentistas no SUS foi uma variável estatisticamente significativa para todos os tipos de procedimentos; por exemplo, municípios com menos dentistas por habitantes (primeiro quintil) tinham 3,23 (IC95\%: 1,85; 5,61) vezes mais chances de não ter nenhum procedimento básico. O porte populacional municipal foi a variável mais significativa para predizer se um município teria algum procedimento de qualquer natureza. Por exemplo, os municípios com menos de 10 mil habitantes tinham 120,2 vezes (IC95\%: 85,1; $169,9)$ mais chances de não ter nenhum procedimento endodôntico se comparados a municípios com mais de 50 mil habitantes.

Foi avaliada a exclusão de alguns valores extremos: taxa de dentistas $>20$ por $10 \mathrm{mil} / \mathrm{ha}$ bitantes-ano, taxa de equipos $>15$ por $10 \mathrm{mil} /$ habitantes-ano e cobertura de planos privados $>50 \%$. A exclusão desses valores não alterou as médias das variáveis e a exclusão conjunta de 114 municípios com ao menos um desses valores alterou os coeficientes de regressão somente na terceira casa decimal.

\section{Discussão}

Os resultados deste estudo mostram que há diferença de produtividade dos municípios com 
Tabela 1

Descrição das taxas de procedimentos odontológicos especializados e características de serviços de saúde dos municípios brasileiros ( $\mathrm{N}=5.564$ ) em 2010 .

\begin{tabular}{|c|c|c|c|c|c|}
\hline & Média & DP & Mínimo & Mediana & Máximo \\
\hline Taxa de procedimentos básicos por 10 mil habitantes-ano, 2010 (SIA-SUS) & $10.686,7$ & $60.240,8$ & 0,0 & $5.545,7$ & $3.209 .406,0$ \\
\hline Taxa de procedimentos de periodontia por 10 mil habitantes-ano, 2010 (SIA-SUS) & 75,3 & 322,9 & 0,0 & 0,0 & $7.409,8$ \\
\hline Taxa de procedimentos de endodontia por 10 mil habitantes-ano, 2010 (SIA-SUS) & 55,7 & 336,6 & 0,0 & 0,0 & $8.848,9$ \\
\hline Taxa de procedimentos de cirurgia oral por 10 mil habitantes-ano, 2010 (SIA-SUS) & 292,9 & $7.753,8$ & 0,0 & 31,5 & $575.793,9$ \\
\hline Despesa anual empenhada em saúde/habitantes em R\$, 2010 (indicador 2.1 do SIOPS) & 388,4 & 184,4 & 0,0 & 348,4 & $2.029,9$ \\
\hline Cobertura de planos odontológicos privados em \% de habitantes, 2010 (ANS-SIB) & 1,4 & 3,8 & 0,0 & 0,2 & 100,0 \\
\hline Cobertura de ESB-ESF em \% de habitantes, 2010 (SIAB) & 62,5 & 36,0 & 0,0 & 70,9 & 100,0 \\
\hline Taxa de cirurgiões-dentistas trabalhando no SUS por 10 mil habitantes-ano, 2010 (SIA-SUS) & 5,4 & 3,6 & 0,0 & 4,5 & 48,9 \\
\hline Taxa de equipos odontológicos disponíveis no SUS por 10 mil habitantes-ano, 2010 (CNES) & 3,5 & 2,1 & 0,0 & 3,1 & 27,5 \\
\hline
\end{tabular}

ANS-SIB: Agência Nacional de Saúde Suplementar - Sistema de Informações de Beneficiários; CNES: Cadastro Nacional de Estabelecimentos em Saúde; DP: desvio-padrão; ESB-ESF: equipes de saúde bucal da Estratégia Saúde da Família; SIA-SUS: Sistema de Informações Ambulatoriais; SIAB: Sistema de Informações em Atenção Básica; SIOPS: Sistema de Informações sobre Orçamentos Públicos em Saúde; SUS: Sistema Único de Saúde.

Tabela 2

Taxa de procedimentos odontológicos especializados, por 10 mil habitantes, realizados em municípios brasileiros com e sem Centros de Especialidades Odontológicas (CEO) conforme a classificação pelo número de consultórios odontológicos (tipo I, tipo II e tipo III), em 2010

\begin{tabular}{|c|c|c|c|c|c|c|}
\hline \multirow[t]{2}{*}{ Tipo de CEO } & \multicolumn{3}{|c|}{ Todos os municípios do Brasil } & \multicolumn{3}{|c|}{$\begin{array}{c}\text { Apenas municípios que realizaram } \\
\text { procedimentos }\end{array}$} \\
\hline & Média & +/- DP & $\mathrm{n}$ & Média & +/- DP & $\mathbf{n}$ \\
\hline \multicolumn{7}{|c|}{ Procedimentos endodônticos } \\
\hline Não possui & 44,1 & 342,1 & 4.851 & 265,2 & 803,4 & 807 \\
\hline Tipo I & 160,1 & 392,5 & 279 & 173,8 & 406,1 & 257 \\
\hline Tipo II & 123,6 & 194,7 & 355 & 128,0 & 196,7 & 343 \\
\hline Tipo III & 91,9 & 120,0 & 79 & 94,3 & 120,6 & 77 \\
\hline Total & 55,7 & 336,6 & 5.564 & 208,8 & 627,0 & 1.484 \\
\hline \multicolumn{7}{|c|}{ Procedimentos periodontais } \\
\hline Não possui & 46,1 & 270,5 & 4.851 & 240,7 & 579,5 & 928 \\
\hline Tipo 1 & 270,4 & 406,0 & 279 & 298,2 & 416,5 & 253 \\
\hline Tipo II & 293,4 & 633,2 & 355 & 304,6 & 642,5 & 342 \\
\hline Tipo III & 202,7 & 244,9 & 79 & 208,0 & 245,9 & 77 \\
\hline Total & 75,3 & 323,0 & 5.564 & 261,9 & 560,3 & 1.600 \\
\hline \multicolumn{7}{|c|}{ Procedimentos básicos } \\
\hline Não possui & $10.887,6$ & $63.963,0$ & 4.851 & $11.307,2$ & $65.147,7$ & 4.671 \\
\hline Tipo I & $10.080,9$ & $22.982,3$ & 280 & $10.190,1$ & $23.082,7$ & 277 \\
\hline Tipo II & $9.176,6$ & $23.352,9$ & 355 & $9.228,6$ & $23.408,8$ & 353 \\
\hline Tipo III & $7.235,8$ & $6.007,0$ & 78 & $7.235,8$ & $6.007,0$ & 78 \\
\hline Total & $10.686,6$ & $60.240,8$ & 5.564 & $11.054,2$ & $61.235,0$ & 5.379 \\
\hline \multicolumn{7}{|c|}{ Procedimentos de cirurgia oral menor } \\
\hline Não possui & 274,6 & $8.300,2$ & 4.851 & 370,9 & $9.644,2$ & 3592 \\
\hline Tipo 1 & 426,4 & 542,7 & 280 & 435,7 & 544,9 & 274 \\
\hline Tipo II & 437,1 & 791,6 & 355 & 438,4 & 792,4 & 354 \\
\hline Tipo III & 298,9 & 307,1 & 78 & 298,9 & 307,1 & 78 \\
\hline Total & 293,0 & 7753,8 & 5.564 & 379,3 & $8.820,6$ & 4.298 \\
\hline
\end{tabular}

DP: desvio-padrão. 
Razão de taxas (RT) e intervalo de 95\% de confiança (IC95\%) de procedimentos básicos, de periodontia, de endodontia e de cirurgia oral menor em três modelos de regressão múltipla binomial negativa inflada de zeros para os municípios brasileiros $(\mathrm{N}=5.564)$ em 2010 .

\begin{tabular}{|c|c|c|c|c|c|c|c|c|c|c|c|c|}
\hline & \multicolumn{3}{|c|}{ Endodontia } & \multicolumn{3}{|c|}{ Periodontia } & \multicolumn{3}{|c|}{ Procedimentos básicos } & \multicolumn{3}{|c|}{ Cirurgia oral menor } \\
\hline & RT & IC95\% & $\begin{array}{l}\text { Valor } \\
\text { de } p \text { * }\end{array}$ & RT & IC95\% & $\begin{array}{c}\text { Valor } \\
\text { de } p \text { * }\end{array}$ & RT & IC95\% & $\begin{array}{l}\text { Valor } \\
\text { de } p \text { * }\end{array}$ & RT & IC95\% & $\begin{array}{l}\text { Valor } \\
\text { de } p \text { * }\end{array}$ \\
\hline \multicolumn{13}{|l|}{ Modelo 1} \\
\hline Não possui & 1,00 & & 0,08 & 1,00 & & 0,66 & 1,00 & & 0.02 & 1,00 & & 0,75 \\
\hline Tipo I & 0,92 & 0,$60 ; 1,41$ & & 1,10 & 0,$69 ; 1,73$ & & 0,72 & 0,$54 ; 0,96$ & & 1,19 & 0,$73 ; 1,95$ & \\
\hline Tipo II & 0,79 & 0,$51 ; 1,20$ & & 1,14 & 0,$73 ; 1,78$ & & 0,69 & 0,$53 ; 0,90$ & & 1,20 & 0,$76 ; 1,92$ & \\
\hline Tipo III & 0,55 & 0,$33 ; 0,92$ & & 0,86 & 0,$50 ; 1,47$ & & 0,62 & 0,$45 ; 0,86$ & & 0,94 & 0,$53 ; 1,67$ & \\
\hline \multicolumn{13}{|l|}{ Modelo 2} \\
\hline Não possui & 1,00 & & 0,09 & 1,00 & & 0,09 & 1,00 & & 0,96 & 1,00 & & 0,02 \\
\hline Tipo I & 1,59 & 1,$07 ; 2,37$ & & 1,72 & 1,$10 ; 2,67$ & & 1,07 & 0,$80 ; 1,42$ & & 1,91 & 1,$20 ; 3,02$ & \\
\hline Tipo II & 1,52 & 1,$01 ; 2,28$ & & 1,68 & 1,$09 ; 2,61$ & & 1,06 & 0,$81 ; 1,39$ & & 1,99 & 1,$29 ; 3,08$ & \\
\hline Tipo III & 1,66 & 1,$02 ; 2,69$ & & 1,65 & 0,$97 ; 2,78$ & & 1,08 & 0,$78 ; 1,50$ & & 1,48 & 0,$86 ; 2,55$ & \\
\hline \multicolumn{13}{|l|}{ Modelo 3} \\
\hline Não possui & 1,00 & & 0,02 & 1,00 & & 0,05 & 1,00 & & 0,97 & 1,00 & & $<0,01$ \\
\hline Tipo I & 1,80 & 1,$21 ; 2,68$ & & 1,80 & 1,$15 ; 2,80$ & & 1,06 & 0,$79 ; 1,40$ & & 2,28 & 1,$44 ; 3,61$ & \\
\hline Tipo II & 1,67 & 1,$11 ; 2,50$ & & 1,79 & 1,$14 ; 2,79$ & & 1,07 & 0,$81 ; 1,36$ & & 2,26 & 1,$47 ; 3,48$ & \\
\hline Tipo III & 1,89 & 1,$15 ; 3,10$ & & 1,75 & 1,$04 ; 2,96$ & & 1,06 & 0,$76 ; 1,45$ & & 1,69 & 1,$01 ; 2,86$ & \\
\hline
\end{tabular}

CEO: Centro de Especialidades Odontológicas; Modelo 1: tipo de CEO + tempo de instalação + número de CEO no município; Modelo 2: modelo 1 + \% mulheres $+\%$ adultos $+\%$ idosos + porte municipal $+\%$ habitantes na zona rural; Modelo 3: modelo 2 + Produto Interno Bruto (PIB) municipal per capita + Índice de Desenvolvimento Humano (IDH) municipal + renda média municipal.

* Valor de $\mathrm{p}$ do teste Wald de que todos os coeficientes da mesma variável são iguais.

Nota: variáveis usadas para modelar inflação de zeros (resultados Tabela 4): porte municipal, número de dentistas no Sistema Único de Saúde (SUS) e número de equipes odontológicos disponíveis no SUS.

e sem CEO, todavia essa associação depende do tipo de procedimento analisado. Em relação aos procedimentos básicos realizados pelos CEOs, não existe diferença entre municípios com e sem os CEO. No entanto, para os procedimentos de endodontia, periodontia e cirurgia oral menor, os municípios com centros de especialidade apresentaram taxas maiores. Assim, a presente análise mostra maiores taxas de produção ambulatorial de saúde bucal em municípios com o incentivo para média complexidade no SUS, conforme o modelo 3, o que já era expresso no campo teórico. Por fim, parte da diferença de produtividade não é explicada por fatores relacionados à estrutura e cobertura dos serviços públicos e privados, conforme o modelo 4 .

A presença de maiores taxas de procedimentos especializados nos municípios com CEO pode ser por conta da presença dos centros. Entretanto, o estabelecimento de tal relação é complexo e necessita de estudos que comparem a produtividade dos CEOs com centros de referência existentes em municípios sem CEO. É importante observar que muitos municípios brasileiros já possuíam alta produção de serviços odontológicos especializados oriunda de centros que não foram estabelecidos pelo estímulo do Ministério da Saúde. São escassos os estudos que avaliam a produtividade dos CEOs, mas há relatos de que a maioria deles não consegue produzir o suficiente para atingir as metas preconizadas 5,9. Além disso, não se conhecem fatores relacionados à capacidade dos centros em atingir metas. Paradoxalmente, há relatos que os CEO apresentam mais facilidade em atingir metas de produção para procedimentos de atenção básica ${ }^{9}$, porém, no contexto municipal do presente trabalho, tal produção não foi significativa. Uma explicação é que esses procedimentos já possuíam alta produção na atenção básica, não sendo alterada pela produção dos CEOs.

No Brasil, a baixa oferta de serviços odontológicos de média complexidade tem sido reconhecida por gestores municipais 13 , desse modo os centros de especialidades odontológicas fazem parte da expansão do acesso aos serviços especializados, sendo evidenciado neste estudo quando houve o controle por um maior número 
Razão de chances (OR) e intervalo de 95\% de confiança (IC95\%) de um município não realizar procedimento básico, de periodontia, de endodontia ou de cirurgia oral menor no modelo 4 de regressão múltipla binomial negativa inflada de zeros para os municípios brasileiros ( $\mathrm{N}=5.564)$ em 2010 .

\begin{tabular}{|c|c|c|c|c|c|c|c|c|}
\hline \multirow[t]{2}{*}{ Variável/Categoria } & \multicolumn{2}{|c|}{$\begin{array}{c}\text { Procedimentos } \\
\text { básicos }\end{array}$} & \multicolumn{2}{|c|}{ Endodontia } & \multicolumn{2}{|c|}{ Periodontia } & \multicolumn{2}{|c|}{ Cirurgia oral menor } \\
\hline & OR & IC95\% & OR & IC95\% & OR & IC95\% & OR & IC95\% \\
\hline \multicolumn{9}{|c|}{ Porte populacional municipal (por mil habitantes) } \\
\hline$>50$ & 1,00 & & 1,00 & & 1,00 & & 1,00 & \\
\hline $40|-| 50$ & 2,22 & 0,$56 ; 8,82$ & 3,23 & 2,$02 ; 5,16$ & 3,76 & 2,$24 ; 6,31$ & 0,01 & 0,$00 ;>1,00$ \\
\hline $30 \mid-40$ & 1,75 & 0,$54 ; 5,62$ & 7,56 & 5,$21 ; 10,98$ & 9,53 & 6,$31 ; 14,39$ & $>1,00$ & 0,$00 ;>1,00$ \\
\hline $20 \mid-30$ & 1,72 & 0,$63 ; 4,71$ & 11,71 & 8,$34 ; 16,45$ & 14,01 & 9,$57 ; 20,50$ & $>1,00$ & 0,$00 ;>1,00$ \\
\hline 10|-20 & 4,25 & 1,$87 ; 9,67$ & 34,62 & 24,$94 ; 48,06$ & 34,64 & 24,$00 ; 49,99$ & $>1,00$ & 0,$00 ;>1,00$ \\
\hline $0-10$ & 7,96 & 3,$55 ; 17,88$ & 120,23 & 85,$06 ; 169,94$ & 117,54 & 80,$22 ; 172,22$ & $>1,00$ & 0,$00 ;>1,00$ \\
\hline \multicolumn{9}{|c|}{ Taxa de dentistas no SUS (por mil habitantes) } \\
\hline Quintil 5 (maior) & 1,00 & & 1,00 & & 1,00 & & 1,00 & \\
\hline Quintil 4 & 1,13 & 0,$65 ; 1,98$ & 2,21 & 1,$74 ; 2,79$ & 1,93 & 1,$53 ; 2,44$ & 1,57 & 1,$04 ; 2,35$ \\
\hline Quintil 3 & 1,21 & 0,$69 ; 2,12$ & 4,11 & 3,$18 ; 5,32$ & 3,42 & 2,$66 ; 4,39$ & 1,92 & 1,$28 ; 2,88$ \\
\hline Quintil 2 & 2,09 & 1,$22 ; 3,60$ & 7,87 & 5,$93 ; 10,44$ & 5,87 & 4,$47 ; 7,70$ & 3,35 & 2,$25 ; 4,99$ \\
\hline Quintil 1 (menor) & 3,23 & 1,$85 ; 5,61$ & 19,37 & 13,$99 ; 26,82$ & 13,13 & 9,$60 ; 17,98$ & 6,57 & 4,$33 ; 9,96$ \\
\hline \multicolumn{9}{|c|}{ Taxa de equipes odontológicos no SUS (por mil } \\
\hline \multicolumn{9}{|l|}{ habitantes) } \\
\hline Quintil 1 (menor) & 1,00 & & 1,00 & & 1,00 & & 1,00 & \\
\hline Quintil 2 & 0,50 & 0,$31 ; 0,79$ & 1,26 & 0,$97 ; 1,65$ & 0,96 & 0,$74 ; 1,26$ & 0,76 & 0,$52 ; 1,11$ \\
\hline Quintil 3 & 0,39 & 0,$23 ; 0,66$ & 1,10 & 0,$83 ; 1,44$ & 0,85 & 0,$65 ; 1,12$ & 0,71 & 0,$48 ; 1,05$ \\
\hline Quintil 4 & 0,52 & 0,$31 ; 0,88$ & 1,24 & 0,$93 ; 1,65$ & 0,98 & 0,$73 ; 1,30$ & 0,90 & 0,$61 ; 1,33$ \\
\hline Quintil 5 (maior) & 0,62 & 0,$37 ; 1,06$ & 1,45 & 1,$07 ; 1,97$ & 1,20 & 0,$88 ; 1,62$ & 1,10 & 0,$74 ; 1,62$ \\
\hline
\end{tabular}

SUS: Sistema Único de Saúde.

Nota: resultados do processo de modelagem de taxas para municípios diferentes de zero são apresentados na Tabela 5.

de variáveis de confusão (modelo 3 da regressão). Um estudo mostrou que, dentre as pessoas que não obtiveram atendimento odontológico, a falta de especialistas e de equipamentos foram responsáveis por, respectivamente, 3,5\% e 9,6\% dos casos, enquanto $46 \%$ não o obtiveram por falta de vagas 14 . A falta de recursos humanos também foi referida como um entrave na implantação das políticas de saúde bucal em Salvador 15. Tais fatores podem ser considerados como indicadores de estrutura na concepção de Donabedian 16 e podem ter impacto em indicadores de processo (i.e., taxas de procedimentos). Reconhece-se que um mínimo de estrutura é necessário, mas não suficiente para garantir cuidados de elevada qualidade 16,17. Com base neste estudo, verifica-se que os municípios com maior taxa de cirurgiões-dentistas no SUS possuíam maiores taxas para qualquer tipo de procedimento. Por outro lado, outras variáveis de estrutura, como equipos/habitantes, não apresentaram um padrão claro de associação. É possível que o dentista seja o fator mais crítico no processo de trabalho odontológico.

Das variáveis socioeconômicas estudadas, municípios com habitantes de maior renda média apresentaram maiores taxas para quaisquer procedimentos. No Brasil, ainda há injustas desigualdades no acesso aos serviços odontológicos 14,18 e as tendências mostram que reduções nas desigualdades de acesso podem ter sido fruto de melhorias nas condições de saúde bucal e não de aumento de acesso 18,19. Considerando que o impacto de políticas públicas pode favorecer os mais ricos 20,21, é fundamental que a Política Nacional de Saúde Bucal, ao mesmo tempo em que dê acesso a todas as classes sociais, garanta mais acesso a quem possui maior nível de necessidades (clínicas), promovendo equidade vertical e horizontal 22.

A avaliação da produção municipal traz implícita uma avaliação da integração dos CEO no sistema municipal. Estudos mostram que o aumento da cobertura de saúde da família estaria 
Tabela 5

Razão de taxas (RT) e intervalo de 95\% de confiança (IC95\%) de procedimentos básicos, de periodontia, de endodontia e de cirurgia oral menor no modelo 4 de regressão múltipla binomial negativa inflada de zeros para os municípios brasileiros ( $N=5.564)$ em 2010.

\begin{tabular}{|c|c|c|c|c|c|c|c|c|c|c|c|c|}
\hline & \multicolumn{3}{|c|}{ Endodontia * } & \multicolumn{3}{|c|}{ Periodontia * } & \multicolumn{3}{|c|}{ Procedimentos básicos * } & \multicolumn{3}{|c|}{ Cirurgia oral menor * } \\
\hline & RT & IC95\% & $\begin{array}{c}\text { Valor } \\
\text { de } p * \star\end{array}$ & RT & IC95\% & $\begin{array}{c}\text { Valor } \\
\text { de } p * \star\end{array}$ & RT & IC95\% & $\begin{array}{c}\text { Valor } \\
\text { de } p^{\star \star}\end{array}$ & RT & IC95\% & $\begin{array}{c}\text { Valor } \\
\text { de } p^{\star *}\end{array}$ \\
\hline \multicolumn{13}{|l|}{ Tipo de CEO } \\
\hline Não possui & 1,00 & & 0,02 & 1,00 & & 0,11 & 1,00 & & 0,68 & 1,00 & & 0,07 \\
\hline Tipo 1 & 1,74 & 1,$15 ; 2,62$ & & 1,74 & 1,$10 ; 2,75$ & & 0,85 & 0,$64 ; 1,12$ & & 1,77 & 1,$13 ; 2,80$ & \\
\hline Tipo II & 1,73 & 1,$14 ; 2,62$ & & 1,69 & 1,$09 ; 2,66$ & & 0,87 & 0,$66 ; 1,12$ & & 1,71 & 1,$11 ; 2,63$ & \\
\hline Tipo III & 2,08 & 1,$26 ; 3,44$ & & 1,60 & 0,$94 ; 2,73$ & & 0,87 & 0,$62 ; 1,17$ & & 1,33 & 0,$79 ; 2,20$ & \\
\hline $\begin{array}{l}\text { Cobertura de } \\
\text { planos privados } \\
\text { odontológicos }\end{array}$ & & & & & & & & & & & & \\
\hline Quintil 1 (menor) & 1,00 & & 0,01 & 1,00 & & 0,05 & 1,00 & & $<0,01$ & 1,00 & & $<0,01$ \\
\hline Quintil 2 & 0,67 & 0,$46 ; 0,95$ & & 0,58 & 0,$40 ; 0,83$ & & 0,94 & 0,$85 ; 1,03$ & & 0,53 & 0,$44 ; 0,64$ & \\
\hline Quintil 3 & 0,63 & 0,$44 ; 0,90$ & & 0,63 & 0,$44 ; 0,91$ & & 1,08 & 0,$98 ; 1,20$ & & 0,54 & 0,$44 ; 0,65$ & \\
\hline Quintil 4 & 0,52 & 0,$36 ; 0,77$ & & 0,60 & 0,$41 ; 0,87$ & & 0,98 & 0,$88 ; 1,06$ & & 0,60 & 0,$51 ; 0,76$ & \\
\hline Quintil 5 (maior) & 0,46 & 0,$30 ; 0,70$ & & 0,64 & 0,$42 ; 0,98$ & & 0,88 & 0,$78 ; 0,99$ & & 0,49 & 0,$41 ; 0,64$ & \\
\hline $\begin{array}{l}\text { Despesas totais em } \\
\text { saúde per capita } \\
\text { [em R\$, 2010] } \\
\text { (SIOPS) }\end{array}$ & & & & & & & & & & & & \\
\hline Quintil 1 (menor) & 1,00 & & 0,32 & 1,00 & & 0,14 & 1,00 & & $<0,01$ & 1,00 & & $<0,01$ \\
\hline Quintil 2 & 1,28 & 0,$99 ; 1,66$ & & 1,20 & 0,$92 ; 1,56$ & & 1,12 & 1,$02 ; 1,24$ & & 1,44 & 1,$22 ; 1,69$ & \\
\hline Quintil 3 & 1,24 & 0,$94 ; 1,62$ & & 1,41 & 1,$07 ; 1,86$ & & 1,22 & 1,$10 ; 1,35$ & & 1,45 & 1,$22 ; 1,72$ & \\
\hline Quintil 4 & 1,16 & 0,$88 ; 1,53$ & & 1,07 & 0,$81 ; 1,42$ & & 0,96 & 0,$85 ; 1,06$ & & 1,64 & 1,$35 ; 1,98$ & \\
\hline Quintil 5 (maior) & 1,29 & 0,$95 ; 1,77$ & & 1,17 & 0,$84 ; 1,63$ & & 1,27 & 1,$12 ; 1,44$ & & 2,81 & 2,$24 ; 3,51$ & \\
\hline $\begin{array}{l}\text { Cobertura de } \\
\text { equipes de saúde } \\
\text { bucal no município }\end{array}$ & & & & & & & & & & & & \\
\hline Quintil 1(menor) & 1,00 & & 0,09 & 1,00 & & 0,01 & 1,00 & & $<0,01$ & 1,00 & & $<0,01$ \\
\hline Quintil 2 & 0,79 & 0,$63 ; 1,01$ & & 1,26 & 0,$98 ; 1,61$ & & 1,38 & 1,$25 ; 1,51$ & & 1,12 & 0,$94 ; 1,32$ & \\
\hline Quintil 3 & 0,70 & 0,$53 ; 1,91$ & & 1,10 & 0,$83 ; 1,46$ & & 1,59 & 1,$43 ; 1,76$ & & 1,30 & 1,$08 ; 1,57$ & \\
\hline Quintil 4 & 0,69 & 0,$51 ; 0,93$ & & 1,35 & 0,$98 ; 1,87$ & & 1,18 & 1,$06 ; 1,30$ & & 0,91 & 0,$75 ; 1,10$ & \\
\hline Quintil 5 (maior) & 0,77 & 0,$56 ; 1,06$ & & 1,69 & 1,$22 ; 2,36$ & & 1,49 & 1,$34 ; 1,66$ & & 1,33 & 1,$08 ; 1,63$ & \\
\hline $\begin{array}{l}\text { Taxa de dentistas } \\
\text { no SUS (por } 10 \text { mil } \\
\text { habitantes) }\end{array}$ & & & & & & & & & & & & \\
\hline Quintil 1 (menor) & 1,00 & & 0,07 & 1,00 & & $<0,01$ & 1,00 & & $<0,01$ & 1,00 & & $<0,01$ \\
\hline Quintil 2 & 1,52 & 1,$13 ; 2,04$ & & 1,45 & 1,$06 ; 1,97$ & & 1,11 & 1,$00 ; 1,23$ & & 1,06 & 0,$87 ; 1,27$ & \\
\hline Quintil 3 & 1,16 & 0,$86 ; 1,58$ & & 1,14 & 0,$83 ; 1,55$ & & 1,30 & 1,$18 ; 1,49$ & & 1,18 & 0,$98 ; 1,44$ & \\
\hline Quintil 4 & 1,24 & 0,$90 ; 1,70$ & & 1,13 & 0,$82 ; 1,57$ & & 1,79 & 1,$59 ; 1,99$ & & 1,56 & 1,$25 ; 1,91$ & \\
\hline Quintil 5 (maior) & 1,37 & 0,$96 ; 1,98$ & & 1,72 & 1,$19 ; 2,48$ & & 1,87 & 1,$65 ; 2,08$ & & 1,31 & 1,$04 ; 1,63$ & \\
\hline
\end{tabular}

(continua) 


\begin{tabular}{|c|c|c|c|c|c|c|c|c|c|c|c|c|}
\hline & \multicolumn{3}{|c|}{ Endodontia * } & \multicolumn{3}{|c|}{ Periodontia * } & \multicolumn{3}{|c|}{ Procedimentos básicos * } & \multicolumn{3}{|c|}{ Cirurgia oral menor * } \\
\hline & RT & IC95\% & $\begin{array}{c}\text { Valor } \\
\text { de } p^{\star \star}\end{array}$ & RT & $\mathrm{IC} 95 \%$ & $\begin{array}{c}\text { Valor } \\
\text { de } p \text { ** }\end{array}$ & RT & IC95\% & $\begin{array}{c}\text { Valor } \\
\text { de } p^{\star \star}\end{array}$ & RT & IC95\% & $\begin{array}{c}\text { Valor } \\
\text { de } p \text { ** }\end{array}$ \\
\hline \multicolumn{13}{|l|}{ Taxa de equipes } \\
\hline \multicolumn{13}{|l|}{ odontológicos no } \\
\hline \multicolumn{13}{|l|}{ SUS (por $10 \mathrm{mil}$} \\
\hline \multicolumn{13}{|l|}{ habitantes) } \\
\hline Quintil 1(menor) & 1,00 & & $<0,01$ & 1,00 & & 0,06 & 1,00 & & $<0,01$ & 1,00 & & $<0,01$ \\
\hline Quintil 2 & 0,82 & 0,$65 ; 1,06$ & & 0,71 & 0,$55 ; 0,91$ & & 0,86 & 0,$78 ; 0,94$ & & 0,64 & 0,$53 ; 0,75$ & \\
\hline Quintil 3 & 1,02 & 0,$76 ; 1,36$ & & 0,93 & 0,$70 ; 1,24$ & & 1,53 & 1,$36 ; 1,69$ & & 0,78 & 0,$64 ; 0,94$ & \\
\hline Quintil 4 & 1,12 & 0,$80 ; 1,58$ & & 0,85 & 0,$61 ; 1,18$ & & 0,93 & 0,$82 ; 0,82$ & & 0,99 & 0,$81 ; 1,23$ & \\
\hline Quintil 5 (maior) & 1,73 & 1,$17 ; 2,54$ & & 0,94 & 0,$64 ; 1,38$ & & 1,12 & 1,$01 ; 1,27$ & & 1,14 & 0,$90 ; 1,44$ & \\
\hline
\end{tabular}

CEO: Centro de Especialidades Odontológicas; SIOPS: Sistema de Informações sobre Orçamentos Públicos em Saúde; SUS: Sistema Único de Saúde.

* Ajustado por: tipo de CEO (o mais antigo do município), tempo de instalação do CEO mais antigo, número de CEO no município, \% mulheres, \% adultos,

\% idosos, porte populacional municipal, \% habitantes na zona rural, Produto Interno Bruto (PIB) municipal per capita, renda média dos indivíduos do município, Índice de Desenvolvimento Humano (IDH) municipal, todas as variáveis da coluna;

** Valor de $\mathrm{p}$ do teste Wald de que todos coeficientes da mesma variável são iguais.

Nota: variáveis usadas para modelar o processo de inflação de zeros (resultados Tabela 4): porte municipal, número de dentistas no SUS e número de equipos odontológicos disponíveis no SUS

associado ao aumento de referência para serviços especializados ${ }^{6}$. No presente estudo, aumento da cobertura de ESB-ESF não esteve associado a procedimentos de endodontia. Isso pode sinalizar problemas específicos no sistema de referenciamento, como problemas de tempo de espera, distâncias geográficas entre os serviços e diálogo/relacionamento entre profissionais da atenção básica e especializada 23,24,25.

O presente trabalho possui um caráter inédito e traz importantes contribuições, mesmo com limitações. Um aspecto positivo é a realização de análises englobando todos os centros cadastrados, configurando-se num censo livre de vieses de seleção. A avaliação em nível municipal permite avaliar a contribuição dos CEO dentro do sistema municipal. Em terceiro, foram utilizadas bases de dados oficiais que são referências para os gestores planejarem as políticas públicas. Além disso, foi possível avaliar o efeito de fatores que poderiam confundir os resultados ou mesmo ser mediadores. Como limitações, esse estudo usou dados secundários, que podem apresentar sub ou supernotificação. Entretanto, acredita-se que essa limitação não esteja associada à presença/ausência de CEO e se sabe que algumas bases, como o SIOPS, possuem boa validade 26 . Outra limitação é que este estudo considerou a data de habilitação e não a data do início das atividades dos CEO; logo, as unidades descredenciadas ou fora de funcionamento foram consideradas ativas. Por fim, uma limitação importante é em relação à dificuldade de estabelecimento de relação causal. Estudos com desenho longitudinal, utilizando séries temporais, devem levar em consideração as tendências existentes de redução de determinados procedimentos como extrações e restaurações 27.

Em conclusão, mostrou-se que os CEO parecem ter um efeito positivo na produção municipal de procedimentos especializados, sobretudo para endodontia. Todavia, as análises mostraram que vários outros fatores também são essenciais e podem explicar as associações observadas. 


\section{Resumen}

El objetivo fue describir la producción de los servicios especializados en los municipios brasileños con y sin Centros Especializados Dentales (CEDs) y estudiar los factores asociados. Los datos fueron recogidos a partir de los siguientes sistemas: Departamento de Informática del SUS, Atlas del Desarrollo Humano y el Instituto Brasileño de Geografía y Estadística. Fueron utilizadas regresiones binomiales negativas infladas con ceros para ajustar las tasas de los procedimientos de endodoncia, atención primaria, periodoncia y cirugía. Después de controlar los factores sociodemográficos, los municipios con CEDs presentan tasas más altas que los sin CEDs, excepto para la tasa de atención primaria. En el modelo final, incluyendo los factores de la estructura de servicio, municipios con CEDs tipo III mostraron una tasa de procedimientos de endodoncia 2,08 (IC95\%: $1,26 ; 3,44)$ veces mayor. Entre estos factores, los municipios con mayor gasto en salud y mayores tasas de dentistas en el sistema público se asociaron con tasas más altas de todos los procedimientos. Se concluye que los centros parecen tener un efecto positivo en la producción ambulatoria, principalmente para endodoncia y este efecto no se explica por las variables de estructura.

Servicios de Salud Dental; Políticas de Salud; Atención Secundaria de Salud; Estudios Ecológicos

\section{Colaboradores}

R. K. Celeste participou da concepção do estudo, revisão de literatura, coleta dos dados, análise e interpretação dos resultados e redação do manuscrito. F. R. R Moura, C. P. Santos e M. F. Tovo colaboraram na concepção do estudo, revisão de literatura, coleta dos dados, interpretação dos resultados e redação do manuscrito.

\section{Referências}

1. Pucca Jr. GA, Costa JF, Chagas LD, Sivestre RM Oral health policies in Brazil. Braz Oral Res 2009; 23 Suppl 1:9-16.

2. Pedrazzi V, Dias KR, Rode SM. Oral health in Brazil. Part II: dental specialty centers (CEOs). Braz Oral Res 2008; 22 Suppl 1:18-23.

3. Paim J, Travassos C, Almeida C, Bahia L, Macinko J. The Brazilian health system: history, advances, and challenges. Lancet 2011; 377:1778-97.

4. Pucca Jr. GA, Lucena EH, Cawahisa PT. Financing national policy on oral health in Brazil in the context of the Unified Health System. Braz Oral Res 2010; 24 Suppl 1:26-32.

5. Figueiredo N, Goes PS. Construção da atenção secundária em saúde bucal: um estudo sobre os Centros de Especialidades Odontológicas em Pernambuco, Brasil. Cad Saúde Pública 2009; 25:259-67.

6. Chaves SC, Barros SG, Cruz DN, Figueiredo AC, Moura BL, Cangussu MC. Brazilian Oral Health Policy: factors associated with comprehensiveness in health care. Rev Saúde Pública 2010; 44:1005-13.
7. Lima AC, Cabral ED, Vasconcelos MM. Satisfação dos usuários assistidos nos Centros de Especialidades Odontológicas do Município do Recife, Pernambuco, Brasil. Cad Saúde Pública 2010; 26:9911002.

8. Chaves SCL, Cruz DN, Barros SG, Figueiredo AL Avaliação da oferta e utilização de especialidades odontológicas em serviços públicos de atenção secundária na Bahia, Brasil. Cad Saúde Pública 2011; 27:143-54.

9. Goes PSA, Figueiredo N, Neves JC, Silveira FMM, Costa JFR, Pucca Júnior GA, et al. Avaliação da atenção secundária em saúde bucal: uma investigação nos centros de especialidades do Brasil. Cad Saúde Pública 2012; 28 Suppl:S81-9.

10. Programa das Nações Unidas para Desenvolvimento. Atlas do Desenvolvimento Humano no Brasil. Brasília: Programa das Nações Unidas para Desenvolvimento; 2003. 
11. Ministério da Saúde. Portaria no 2.898 de 2010. Atualiza o anexo da Portaria no 600/GM, de 23 de março de 2006. Diário Oficial da União 2010; 21 set.

12. Ministério da Saúde. Portaria no 599 de 2006. Define a implantação dos Centros de Especialidades Odontológicas (CEO), de Laboratórios Regionais de Prótese Dentária (LRPD) e estabelece normas, critérios e requisitos para o seu credenciamento. Diário Oficial da União 2006; 23 mar.

13. Leal RB, Tomita NE. Assistência odontológica e universalização: percepção de gestores municipais. Ciênc Saúde Coletiva 2006; 11:155-60.

14. Peres KG, Peres MA, Boing AF, Bertoldi AD, Bastos JL, Barros AJ. Reduction of social inequalities in utilization of dental care in Brazil from 1998 to 2008. Rev Saúde Pública 2012; 46:250-8.

15. Soares CLM, Paim JS. Aspectos críticos para a implementação da política de saúde bucal no Município de Salvador, Bahia, Brasil. Cad Saúde Pública 2011; 27:966-74.

16. Donabedian A. Evaluating the quality of medical care. Milbank Mem Fund Q 1966; 44:166-206.

17. Campbell SM, Roland MO, Buetow SA. Defining quality of care. Soc Sci Med 2000; 51:1611-25.

18. Celeste RK, Nadanovsky P, Fritzell J. Trends in socioeconomic disparities in the utilization of dental care in Brazil and Sweden. Scand J Public Health 2011; 39:640-8.

19. Celeste RK, Nadanovsky P, Fritzell J. Trends in socioeconomic disparities in oral health in Brazil and Sweden. Community Dent Oral Epidemiol 2011; 39:204-12.

20. Celeste RK, Nadanovsky P. How much of the income inequality effect can be explained by public policy? Evidence from oral health in Brazil. Health Policy 2010; 97:250-8.
21. Victora CG, Wagstaff A, Schellenberg JA, Gwatkin D, Claeson M, Habicht JP. Applying an equity lens to child health and mortality: more of the same is not enough. Lancet 2003; 362:233-41.

22. Starfield B. Improving equity in health: a research agenda. Int J Health Serv 2001; 31:545-66.

23. Morris AJ, Burke FJ. Primary and secondary dental care: how ideal is the interface? Br Dent J 2001; 191:666-70.

24. Morris AJ, Burke FJ. Primary and secondary dental care: the nature of the interface. Br Dent J 2001; 191:660-4.

25. Barnes JJ, Patel S, Mannocci F. Why do general dental practitioners refer to a specific specialist endodontist in practice? Int Endod J 2011; 44:21-32.

26. Lima CRA, Carvalho MS, Schramm JMA. Financiamento público em saúde e confiabilidade dos bancos de dados nacionais. Um estudo dos anos de 2001 e 2002. Cad Saúde Pública 2006; 22:1855-64.

27. Celeste RK, Vital JF, Junger WL, Reichenheim ME. Séries de procedimentos odontológicos realizadas nos serviços públicos brasileiros, 1994-2007. Ciênc Saúde Coletiva 2011; 16:4523-32.

Recebido em 11/Abr/2013

Versão final reapresentada em 12/Set/2013

Aprovado em 27/Set/2013 